\title{
DOCTRINA
}

\section{Profesión legal y tecnologías de la información y las comunicaciones: Una discusión necesaria}

\author{
Legal profession and information and communication technologies: \\ A necessary discussion
}

Johann S. Benfeld E.

Pontificia Universidad Católica de Valparaíso, Chile

\begin{abstract}
RESUMEN En este trabajo se afirma que, pese a la creciente importancia que en las últimas décadas han adquirido las tecnologías de la información y las comunicaciones (TIC) para la profesión legal, su estudio y eventual enseñanza se ha limitado en nuestro medio, por una parte, a una utilización básica como recursos pedagógicos y, por otra, a una aproximación dogmático-jurídica del fenómeno (derecho y tecnología), sin advertir el impacto que ellas probablemente han de tener en el mediano y largo plazo como habilidades profesionales exorbitantes al currículo tradicional, pero necesarias para el futuro desempeño laboral.
\end{abstract}

PALABRAS CLAVE Ley de Moore, TIC, profesiones legales, enseñanza y ejercicio del derecho en Chile.

ABSTRACT This paper states that despite the growing importance of the so-called information and communication technologies (ICT) for the legal profession in recent years, its study and eventual teaching has been restricted in our environment, first, in a basic use of this technologies as pedagogical resources, and second, to a dogmatic-juridical approach of the phenomenon (law and technology), without noticing the impact they are likely to have, in the medium and long-term, as professional skills exorbitant to the traditional curriculum, but necessary for future job performance.

KEYWORDS Moore's law, ICT, legal professions, teaching and practice of law in Chile. 


\section{Introducción}

En 1965, Gordon E. Moore publicó en la revista Electronics un artículo de sólo cuatro páginas titulado «Cramming more components onto integrated circuits». Como su nombre indica, el objetivo principal de este escrito era explorar las consecuencias teóricas y prácticas de - poder- introducir más componentes en los circuitos integrados.

Moore afirmaba que existía una relación directa entre el número de componentes de un circuito integrado y sus prestaciones, de manera tal que, a mayor número de componentes en un circuito, mayores serían también sus prestaciones individuales y las del dispositivo electrónico correspondiente:

Los circuitos integrados darán lugar a maravillas como los computadores domésticos, o al menos a terminales conectadas a un computador central, los controles automáticos para automóviles y los equipos personales portátiles de comunicaciones (Moore, 1965: 114).

Moore advirtió, además, que, dado el nivel que había alcanzado la tecnología, los costos asociados a la producción de nuevos y más sofisticados circuitos integrados irían disminuyendo de manera gradual y, con ello, también los costos de adquisición de los dispositivos electrónicos para los cuales fueran fabricados. Así las cosas, progresivamente la tecnología avanzada - en su tiempo, los computadores- estaría cada vez más al alcance de las personas comunes (Moore, 1965: 114).

Sin embargo, no fueron sólo esas ideas las que dieron fama mundial a este artículo, sino la formulación de una regla. Ésta, hoy conocida como la «ley de Moore», afirmaba que el aumento de componentes dentro de un circuito integrado se iría duplicando cada año, mientras que los costos de fabricación irían disminuyendo de forma dramática. En 1975, ante la evidencia empírica, en un encuentro internacional sobre dispositivos electrónicos organizado por el Institute of Electrical and Electronics Engineers (IEEE), el propio Moore morigeró su proyección a un periodo de dos años (Moore, 2006: 72).

Con el tiempo, y en lo que a los circuitos integrados toca, la aseveración de Moore se mostró bastante acertada. Tanto si se desagregan todas las variables consideradas en el rendimiento de un circuito integrado (número de componentes y potencia; capacidad de procesamiento de información y aumento de memoria RAM), como si se atiende a los costos de producción, las proyecciones se ajustan en forma razonable a su estimación (Zukerfeld, 2015: 101-103).

Ahora bien, como la prognosis de Moore se halla de manera indisoluble unida a los avances tecnológicos, muy pronto la expectativa de progresión exponencial de los chips se trasladó a toda la industria de la tecnología y, desde ésta, a las otras actividades productivas. La regla que en un principio buscaba explicar un fenómeno 
particular se convirtió en una suerte de expectativa razonable para todos los sectores de la economía:

Tal vez el aspecto más importante de la ley de Moore es que ésta se ha convertido en un predictor casi universal del crecimiento de toda la industria, que no ha detenido su crecimiento exponencial por más de tres décadas (Schaller, 1997: 55).

Es cierto, por una parte, que no todos los sectores de la economía - y la sociedad en general- se han visto transformados de la forma exponencial que predice la ley de Moore (Yoo, 2015: 94-96); por otra parte, ella por sí sola no puede dar cuenta de las profundas transformaciones que en las últimas décadas ha experimentado el mundo por el desarrollo de las nuevas tecnologías. ${ }^{1}$ Sin embargo, es innegable que una de las características dominantes de nuestro tiempo - por lo menos en las sociedades posindustriales de carácter liberal- es la rapidez con que los dispositivos tecnológicos, las estructuras sociales, las formas de trabajo y cooperación han cambiado. En este nuevo escenario, como nunca antes en la historia de la humanidad, la cuestión a resolver no es tanto cómo adaptarse a los cambios que imponen un nuevo orden social, sino más bien cómo renunciar a la idea misma de orden y estabilidad social en función del cambio, entendido ahora como progreso (innovación), cuya única medida de valuación es el mayor rendimiento de bienes, relaciones y tiempo.

En tal dirección, y teniendo a la vista el escenario propuesto por las proyecciones de Moore, es posible preguntarse: ¿en qué medida las tecnologías de la información y las comunicaciones han impactado en el mundo jurídico, y de qué forma ellas han sido incorporadas en la discusión académica? Para intentar responder estas cuestio-

1. De alguna manera, si bien la ley de Moore puede ser considerada un antecedente necesario para comprender el escenario social actual, no debe ser reverenciada como la razón suficiente que explica su complejidad. Por ello, han surgido otras explicaciones que pretenden dar cuenta de las transformaciones sociales que ha producido el desarrollo de las nuevas tecnologías. Entre ellas cabe destacar la «ley de Metcalfe». Al igual que la propuesta de Moore, no se trata de una verdadera ley, sino de una estimación prospectiva, en este caso, del valor que tiene - matemáticamente- la interacción en una red. El valor de una red de comunicaciones, de acuerdo con este criterio, aumenta de manera proporcional al cuadrado del número de usuarios del sistema. En palabras simples, esto quiere decir que las redes de comunicación, una vez que alcanzan un cierto tamaño, tienen un aumento exponencial de relaciones entre sus usuarios. Esto porque una red de comunicación robusta tiene, por una parte, mayor conectividad y, por otra, más información disponible (Larrosa, 200o: 2). «En todo caso, la ley de Moore y su promesa de que "el número de transistores por centímetro cuadrado en un circuito integrado se duplicaría aproximadamente cada 18 a 24 meses", y la ley de Metcalfe, y su paradigma de que "el valor de la red crece exponencialmente", no necesitan competir para ver cuál de las dos ocupará el primer lugar. Si bien es cierto que se necesitan redes interconectadas, confiables y seguras, también lo es que se necesitan computadores potentes, capaces de soportar las exigentes demandas de aplicaciones en algunos sectores. Al fin de cuentas, las redes son redes de computadores. Y las empresas necesitan tanto de las redes como de los computadores para llegar a más lugares, a más gente, de forma cada vez más confiable y segura» (Patiño Builes, 2014: 58). 
nes, comenzaremos con una breve aclaración de conceptos necesarios para comprender las tecnologías de la información y comunicaciones (TIC), con especial consideración a aquéllas que han cobrado en el último tiempo mayor importancia para la profesión jurídica. Luego, nos referiremos brevemente a los desafíos que se ciernen sobre la profesión legal en la era digital. Hecho lo anterior, revisaremos en qué medida, por una parte, los autores nacionales han mostrado algún tipo de interés por las TIC y, por otra, de qué forma éstas se han incorporado en los planes y programas de estudio de las escuelas de Derecho chilenas.

\section{Las tecnologías de la información y comunicación}

Las tecnologías de la información y comunicación comprenden un conjunto de elementos conexos entre sí de naturaleza material (dispositivos electrónicos) e inmaterial (programas informáticos), que posibilitan el suministro e intercambio de información mediante la telecomunicación de recursos - preeminentemente digitales- que permiten el procesamiento, gestión y almacenamiento de gran cantidad de información en un breve espacio de tiempo (Chen y otros, 2014).

Cuando se piensa en los dispositivos electrónicos materiales involucrados en las TIC, los computadores ocupan un lugar de privilegio. Sin embargo, no son los únicos dispositivos relevantes. Terminales multimedia, smartwatch, smartphones, tablets, consolas de videojuegos, dispositivos de almacenamiento y reproducción de audio y video y todo el conjunto de elementos materiales periféricos que requieren esos dispositivos para su funcionamiento (cables, teclados, pantallas, audífonos) forman parte, entre muchos otros, de los dispositivos e insumos materiales que hacen posible el procesamiento y gestión de grandes volúmenes de información de forma rápida.

Los recursos informáticos involucrados, a su turno, son los softwares, es decir, el conjunto de programas que hacen posible que los dispositivos físicos (hardware) hagan las tareas para las cuales han sido diseñados. Considerando que dentro de los programas se encuentran las llamadas aplicaciones (apps), resulta casi imposible hacer una cuenta siquiera general de los programas informáticos. Con todo, sí es posible ensayar una clasificación de acuerdo con la función específica que desempeñan. En este sentido, se habla de software de sistema, de aplicación y de programación. Dentro de los primeros se ubican los sistemas operativos (Windows, Unix, Mac OS, Linux, Chrome OS, entre otros); los programas de sistema son los que permiten la comunicación entre el usuario y el hardware, lo cual hace posible la incorporación de otros softwares particulares destinados a llevar a cabo tareas específicas. Los programas de aplicación son innumerables, y se pueden funcionar de manera independiente o como un conjunto de programas (suite de oficina). A su vez, las aplicaciones pueden estar diseñadas para ser usadas de forma autónoma en el dispositivo computacional (offline), o estar pensadas para su uso en red (intranet o internet). 
Por último, existen los softwares de programación, que permiten - como su nombre lo indica- a programadores, analistas e ingenieros informáticos crear o modificar programas mediante el uso de un lenguaje de programación: Java, C, C++, Python, C\#, Visual Basic .NET, JavaScript, PHP, Perl y Assembly language (ASL) son algunos de estos programas.

\section{Internet como espacio de convergencia}

Si bien es cierto que hardware y software son elementos necesarios en la comprensión del desarrollo de las TIC (Acosta, Miquilena y Riveros, 2014: 15-20), ellos por sí solos no logran explicar su importancia actual. Para hacerlo, hay que atender al ámbito de convergencia en que software y hardware desarrollan todas sus potencialidades y al producto mismo de dicha convergencia. Nos referimos a internet y al advenimiento de la era digital. ${ }^{2}$

Internet, por decirlo de alguna manera, opera como el espacio (virtual) de convergencia comunicacional que, desde la década de los setenta en adelante, viene produciendo una verdadera revolución sociocultural. Al usar la palabra «revolución», no queremos tan sólo hacer uso de un término semántica e ideológicamente fuerte con una finalidad retórica. Más bien, queremos decir que internet es el elemento disruptivo que, con el correr del tiempo, tendrá el poder de explicar los acontecimientos más relevantes de nuestra era:

Historiadores y expertos en política internacional pueden no querer escuchar esto, pero todas las cosas sobre las que ellos enseñan o escriben acerca de geopolítica del siglo XIX son meras notas a pie de página de la Revolución Industrial. Y exactamente lo mismo es probable que sea verdadero cuando nosotros -o nuestros descendientes robots- escriban la historia de la revolución digital del siglo XXI. ${ }^{3}$

2. Es probable que el fenómeno de internet sea el componente más característico de la llamada «era digital», «Tercera (o Cuarta) Revolución Industrial», «sociedad del conocimiento y la información», etcétera. Como se sabe, internet es una interconexión de redes parciales, en la cual se intercambian datos en forma de paquetes digitalizados (TCP/IP) entre computadores. La «universalidad» y la «medialidad del computador» se transforma en una propiedad de la misma red. Internet no está sólo por eso predestinado - ya sea como tal, como World Wide Web (WWW) o de otra forma- a extenderse globalmente, sino también a desarrollar todas las intersecciones y funciones inimaginables, a unir todas las aplicaciones posibles, así como a integrar cada programa: desde el simple e-mail (correo electrónico), el e-banking (banca electrónica), e-learning (aprendizaje a distancia), e-voting (voto electrónico) y otros, pasando por la administración de las casas inteligentes, la ingeniería colaborativa, funcionando las 24 horas del día en todas las zonas horarias del planeta, construyendo proyectos comunes, desarrollando software, en el trabajo editorial y más, hasta la regulación, apoyada por computación, de cadenas integras de producción y de distribución, de ramos enteros y de mercados completos (b2b, b2c, c2c, b2g), etc. (Metzner-Szigeth, 2006).

3. Kevin Drum, «Tech world: Welcome to the digital revolution», Foreign Affairs, julio-agosto de 2018, p. 43, disponible en https://fam.ag/2HRSXco. 
En efecto, sin la Revolución Industrial es poco probable que hubiera surgido una clase media, no se habría incrementado el capitalismo, los procesos de colonización habrían sido aislados - impulsados por la búsqueda de materias primas-e incluso la guerra habría sido más difícil de sustentar sin industrias y acero barato. Pues bien, algo similar podría ocurrir con los fenómenos que en el futuro sean sindicados por los historiadores como característicos de nuestra era.

A diferencia de la Revolución Industrial, la digital no ha impactado tanto en el incremento de bienes materiales de consumo, ${ }^{4}$ sino en algo mucho más radical: la modelación de un nuevo escenario sociocultural, en el cual las categorías espaciotemporales se difuminan - lo mediato da paso a lo inmediato, y lo real a lo virtual-; la comunicación fragmentada se unifica en un sistema multimedial: texto, imagen y sonido conforman un todo comunicacional (Castells, 2005).

En qué medida la revolución digital impactará nuestras sociedades es algo difícil de prever. Aunque es relativamente sencillo advertir las ventajas que la nueva era trae consigo - por lo menos desde un punto de vista económico-, no han faltado quienes denuncian los peligros asociados.

La hiperconectividad, para algunos, diluye las esferas de lo privado y lo público, y con ello también el respeto propio de la individualidad (Han, 2014). El sujeto privado ahora de su centro y de la posibilidad de reflexión se encuentra volcado hacia el estímulo exterior que, en su inconmensurabilidad, le produce una desazón existencial. De ahí que la sociedad de la (hiper) información y el cambio continuo se traduzca en un profundo cansancio del cuerpo social y sus individuos (Han, 2015a).

En este nuevo escenario digital dominado por el big data (Han, 2015b), se afirma, el ejercicio del poder adquiere toda su eficiencia por la sumisión voluntaria de los miembros del cuerpo social, transformados ahora en autosensores de su «desarrollo» $\mathrm{y}$ «éxito» personal, el cual puede ser seguido y controlado por todos y cada uno en el espectro público de la red.

Las anotaciones sobre uno mismo sirven a una ética del yo. ${ }^{5}$ El dataísmo, por el contrario, vacía el self-tracking de toda ética y verdad, y lo convierte en una mera técnica de autocontrol. Los datos recopilados también se publican e intercambian. De esta forma el self-tracking se equipara cada vez más a la autovigilancia. El sujeto de hoy es un empresario de sí mismo. El sujeto explotador de sí mismo se instala en un campo de trabajo en el que es al mismo tiempo víctima y verdugo. En cuanto sujeto que se ilumina y vigila a sí mismo, está aislado en un panóptico en el que es simultáneamente recluso y guardián. El sujeto en red, digitalizado, es un panóptico de sí mismo. Así pues, se delega a cada uno la vigilancia (Han, 2015b: 93).

4. Drum, «Tech world», 44.

5. Aquí se refiere el autor a la antigua práctica de los diarios de vida u obras similares, en que los individuos hacían un ejercicio narrativo para la mejor comprensión de su existencia. 
En este contexto, advierten otros, todo aquello que tenía antes sentido pierde valor y peso. El cambio constante y el deseo desatado de novedad deshilvanan las estructuras sociales y con ello el deseo de persistencia en los compromisos y relaciones. Ahora que las cosas ya no pesan, que han perdido su consistencia y resistencia, estamos en una sociedad líquida. En ésta se han roto los lazos sociales sustantivos, las estructuras de cooperación cuyo sentido era precisamente satisfacer un número finito de necesidades, que han dado paso al crecimiento ilimitado de los deseos reales o supuestos- que dejan siempre al individuo con una abrumadora sensación de vacío (Bauman, 2011):

Y así, la política de «precarización» deliberada llevada a cabo por los operadores de los mercados laborales se ve apoyada e instigada (con sus refuerzos) por las formas de vida, adoptadas deliberadamente o por defecto. Ambas (precarización y forma de vida contemporánea) convergen en un mismo resultado: el desvanecimiento y el marchitamiento, el desmoronamiento y la descomposición de los vínculos humanos, de las comunidades y de las asociaciones. Compromisos como «hasta que la muerte nos separe» se transforman en contratos cuyo contenido es simplemente «hasta que la satisfacción dure». Contratos éstos de naturaleza temporal y transitoria, tanto por diseño como por su impacto pragmático, y, por lo tanto, propensos a dejarse sin efecto unilateralmente, cada vez que uno de los socios advierta una mejor oportunidad y un mayor valor en la exclusión voluntaria de la asociación (Bauman, 2006: 163).

Uno puede, con todo, estar o no de acuerdo con el diagnóstico precedente. Sin embargo, resulta difícil suponer que las relaciones sociales y el mundo tal y como lo conocemos seguirán siendo los mismos luego de la revolución digital. En este sentido, uno de los cambios más importantes que se atisban en el horizonte es el del mercado laboral, debido a los grandes avances que en los últimos años ha experimentado la inteligencia artificial. Esta, constituida por poderosos algoritmos capaces de automatizar tareas físicas e intelectuales, se presenta como una amenaza $-\mathrm{u}$ oportunidad-para las futuras generaciones (Harari, 2016; Susskind, 1996, 2008, 2013).

\section{Importancia e impacto de las TIC para la profesión legal}

Las TIC, unidas a internet, han transformado el mundo contemporáneo. Esta transformación en algunos casos ha resultado bastante evidente —en el ámbito de la entretención masiva y las comunicaciones, por ejemplo-y en otros no tanto, aunque no por ello ha sido menos significativa (algunas de las ventajas, contenidos y operaciones disponibles en la Deep Web, por ejemplo, Echeverri, 2016: 13-49).

El derecho, por cierto, no ha estado ajeno a estos cambios. En tal sentido, es posible hablar de por lo menos tres ámbitos en que las TIC están forzando una revisión tanto de la forma en que se enseña y estudia el derecho como del trabajo legal en 
general. En primer lugar, en los últimos años hemos asistido a un proceso creciente de digitalización de recursos y gestión de los materiales jurídicos tanto en el ámbito público como privado. En el primero, la modernización del Estado ha supuesto el abandono progresivo de las formas tradicionales de buscar, recopilar y gestionar la información - manual y de soporte físico - para dar paso a la incorporación de plataformas que buscan recopilan y gestionar la información de forma digital; ${ }^{6}$ en el segundo, se ha producido un significativo incremento de bases de datos con recursos jurídicos que, de manera progresiva, comienzan a ser utilizadas como herramientas habituales del trabajo legal, ya como recopilaciones masivas de información (Vlex o Westlaw), ya como programas especiales de gestión de oficinas jurídicas (Legal One).

Este proceso acelerado de digitalización de recursos ha encontrado en los últimos años un entorno procesal congenial con los nuevos tiempos gracias a la promulgación de la Ley 20.886, sobre tramitación electrónica. Ésta ha venido a transformar -con grado variable de éxito y alcance- el ejercicio profesional y la labor jurisdiccional (Larroucau, 2017).

Ahora bien, este proceso de digitalización supone, como condición de operatividad, un conocimiento - a lo menos básico- de los operadores jurídicos en orden a buscar, gestionar y producir información relevante en este nuevo escenario. Estos conocimientos básicos y habilidades profesionales se traducen en la idea de «alfabetización digital» (digital literacy), cuyos aspectos medulares se refieren a la capacidad para adquirir conocimientos actualizados y pertinentes para el desempeño profesional en entornos digitales; la capacidad para recoger y analizar información relevante para la práctica profesional a través de dispositivos tecnológicos y medios digitales; la habilidad de trabajar en equipo en entornos digitales (trabajo en red); el conocimiento y manejo de programas, aplicaciones, bases de datos y motores de búsqueda relevantes para la profesión; la capacidad de posicionar y gestionar los productos y marcas legales en la red, etcétera.

En segundo lugar, las nuevas tecnologías y el uso de internet han transformado de manera significativa la forma en que los abogados se relacionan con sus clientes. Ello debido al incremento de la presencia digital de los estudios jurídicos -fenómeno, por cierto, nada nuevo en el mundo desarrollado- y al uso de los medios sociales como canales de expresión, pero también de obtención relevante de información. El uso de plataformas virtuales y el empleo sistemático de redes sociales como elementos habituales del mercado legal, a su vez, está forzando a los estudios jurídicos a trabajar en forma más estrecha con profesionales del mundo de la ingeniería, diseño y medios, de una manera que va mucho más allá de la promoción y publicidad de los servicios jurídicos (Horne, 2014).

6. Sobre los desafíos y expectativas de este programa político en el caso chileno, véase la Agenda Digital 2020, «Chile Digital para tod@s», de 2015, disponible en http://www.agendadigital.gob.cl. 
En tercer y último lugar, el uso progresivo de la inteligencia artificial en el dominio de los servicios jurídicos ha supuesto un cambio significativo en la forma de comprender el «valor» del trabajo legal. En tal sentido, softwares como los ofrecidos por Lemotech (TimeBilling, CaseTracking, IPmanager y otros) han simplificado y masificado las prestaciones jurídicas de naturaleza mecánica, facilitando los procesos y disminuyendo de forma dramática los tiempos de gestión de la información.

Desde la perspectiva de los profesionales del derecho, los nuevos softwares representan una oportunidad de optimización y expansión del mercado. Para ello, es necesario en un nivel inicial conocerlos y saber gestionarlos y, en un nivel avanzado, incluso es deseable comprender su dinámica interna para así poder, en colaboración con otros profesionales de ámbito de la informática, desarrollar nuevos algoritmos que lleven a la inteligencia artificial en el ámbito jurídico a un nuevo nivel (Rostain, Skalbeck y Mulcahy, 2013).

Estos tres ámbitos, entre otros, en que las TIC están forzando una revisión tanto de la forma en que se enseña y estudia el derecho, como del trabajo legal en general (digitalización de recursos y procedimientos jurídicos, existencia de un mercado legal virtual y uso creciente de redes sociales como herramientas de la profesión legal, y aumento progresivo de programas inteligentes en el ámbito del mercado legal) muestran la relevancia e impacto que tienen en la actualidad las tecnologías de la información y las comunicaciones.

La importancia de estas tecnologías para el derecho ha hecho surgir, por lo menos en el mundo anglosajón, una creciente literatura que abarca tanto temas de alfabetización digital para abogados - o futuros abogados-: derecho y tecnología, tecnología legal, y nuevas formas de gestión de los negocios jurídicos (legal management), entre otros. ${ }^{7}$ Más adelante veremos qué tan sensible ha sido la academia en nuestro país frente a las TIC, por lo menos a nivel de la literatura que ésta ha generado.

Ahora bien, debido a la importancia e impacto que en el último tiempo han adquirido las nuevas tecnologías, en una prognosis distópica, algunos ya vaticinan el fin de muchas profesiones y oficios - si no todos- en los próximos años.

El dataísmo adopta un enfoque estrictamente funcional de la humanidad, y tasa el valor de las experiencias humanas según su función en los mecanismos de procesamiento de datos. Si desarrollamos un algoritmo que cumpla mejor la misma función, las experiencias humanas perderán su valor. Así, si podemos sustituir no sólo a taxistas y a médicos, sino también a abogados, a poetas y a músicos con programas informáticos superiores, ¿por qué habría de preocuparnos que dichos programas no

7. En este sentido, y sólo por citar algunos trabajos especializados de carácter general sobre este tema, se puede consultar: Rowland, Kohl y Charlesworth (2017), Gutwirth, Leenes y De Hert (2016), Edwards y Waelde (2000), Lambert (2002), Lim (2002), Phillips (2013) y Reed y Angel (2003). En español, Delgado y Beltrán (2018) y Rincón (2018). 
tengan conciencia ni experiencias subjetivas? Si algún humanista empezara a adular el carácter sagrado de la experiencia humana, los dataístas rechazarían esas bobadas sentimentales. «La experiencia que alabas no es más que un algoritmo bioquímico anticuado. Hace setenta mil años, en la sabana africana, este algoritmo era de última generación. Incluso en el siglo XX era vital para el ejército y para la economía. Pero pronto tendremos algoritmos mucho mejores» (Harari, 2016: 422).

Otros, más optimistas, apuestan más bien por una transformación de la forma del trabajo y no tanto por un reemplazo del factor humano. En esta investigación nos parece que, por ahora, ésta es la posición más razonable de cara al futuro inmediato.

Pero, incluso al ser optimistas, la cuestión sigue siendo cómo han de enfrentar las profesiones en general y la profesión jurídica en particular los nuevos desafíos de la era digital. En otras palabras, ¿cómo han de prepararse los futuros profesionales del derecho para trabajar en un entorno caracterizado por el cambio, la inmediatez y la proscripción de cualquier forma rígida de organización? ¿Cómo han de hacer frente a los desafíos que las nuevas tecnologías representan para el orden jurídico a partir de instituciones y normas pensadas para otro tiempo? ¿Qué habilidades y estrategias deberán usar para ser, a lo menos, tan innovadores como la era demanda? Finalmente, ¿qué insumos han de conocer y saber utilizar los futuros abogados para realizar su cometido en la era digital?

\section{La profesión legal en la era digital}

Sobre el presente y futuro de la profesión legal se ha escrito bastante, sobre todo en el mundo anglosajón. Con todo, no es el objeto de este artículo exponer de forma sistemática el impacto que las nuevas tecnologías están teniendo en las profesiones legales ni responder todas las preguntas que se han formulado en el párrafo precedente. Sin embargo, puede resultar de utilidad decir algunas cosas sobre el particular para poder apreciar en perspectiva la magnitud de los desafíos.

Probablemente una de las voces más autorizadas en esta materia sea la de Richard Susskind, quien desde la década de los noventa viene explorando a través de varios libros el impacto de lo que él denomina «the technology-based internet society» (sociedad de la internet basada en la tecnología) en el estudio y ejercicio de las profesiones legales. Especial mención merecen en este sentido The future of the law (1996), ¿The end of lawyers? (2008), Tomorrow's lawyers (2013) y The future of the professions (2015, coescrito con su hijo Daniel).

Resumiendo mucho las cosas, de acuerdo con este autor, es bastante posible que en un futuro no lejano las nuevas tecnologías reemplacen (substitute) más que complementen (complement) el ejercicio de la profesión legal. En este tránsito de un modelo (el ilustrado industrial) a otro (basado en la tecnología digital), podremos apreciar un conjunto de significativos cambios en la forma de prestar los servicios 
profesionales: primacía del principio de eficiencia por sobre otras prácticas profesionales tradicionales (sujeción a las formas), incorporación de nuevos actores en la prestación de los servicios legales (no necesariamente abogados), progresiva desaparición de los servicios personales de carácter individual (a move away from bespoke service), desagregación de funciones, iteración de trabajo, etcétera.

En el nuevo modelo (the technology-based internet society), la cuestión a resolver será, por tanto, cómo se produce y distribuye el conocimiento y la experiencia dentro de la sociedad. Respecto de esta pregunta caben, según Susskind, siete alternativas, de las cuales la última es la más desconcertante. Estos modelos son el tradicional (centrado en la profesión y sus cultores en un mercado físico); el modelo de trabajo en línea (ejercido por profesionales, pero en un espacio virtual. Los múltiples servicios de asistencia legal en línea son un caso de ello); el modelo paraprofesional (ejercido por personas independientes a la profesión, pero que gracias al conocimiento de algún software pueden proveer los servicios profesionales. Los servicios de declaración de impuestos en línea, por ejemplo); el modelo de la experiencia compartida (aquí es una comunidad pasiva necesitada del servicio, la que se autoinstruye con los insumos informáticos presentes en el sistema. Un sitio web de deudores hipotecarios que comparten información relevante sobre la materia, por ejemplo); el modelo del conocimiento incorporado en el sistema (aquí estamos en presencia de un algoritmo programado para responder de una única y exclusiva forma frente a una exigencia. Los campos obligatorios en ciertos formularios digitales pueden servir de ejemplo, aun cuando se trata de algo más complejo); por último, está el modelo generado por la máquina. Se trata del último caso o alternativa de distribución del conocimiento y experiencia en la sociedad. En este modelo es el propio algoritmo quien genera el conocimiento - de una forma independiente a la humana-; su expresión máxima sería la inteligencia artificial autónoma, aunque, sin llegar aún a ese extremo, ya está presente en múltiples aplicaciones informáticas (Susskind y Susskind, 2015: 215-228). ${ }^{8}$

Todo parece indicar, por lo que venimos diciendo, que la profesión legal se ha de transformar si quiere tener un espacio en la sociedad de la internet. En este sentido, es primordial comprender que los cambios en la profesión legal comienzan desde la formación temprana de los futuros operadores jurídicos (Perlman y Tanenbaum,

8. Respecto de este último modelo es que se han hecho algunos reparos, en cuanto amenaza latente para el futuro de las profesiones. Sin embargo, no es posible saber si el desarrollo eventual de la inteligencia artificial supone una amenaza a las profesiones mismas o, más bien, constituye una alternativa diversa a la forma en que hasta el momento hemos enfrentado el problema de la distribución del conocimiento. En esta dirección, los Susskind hablan de una suerte de falacia de la inteligencia artificial. Ella consiste en concebirla como una versión mejorada de las formas de razonamiento humano, sin advertir que es -o puede ser- una forma de razonamiento no humano, que requiere para su comprensión nuevas categorías de análisis (Susskind y Susskind: 2018, 132-134). En el mismo sentido avanza el documental AlphaGo de Greg Kohs. 
2016). Respecto de dicha formación, no basta con incorporar un cúmulo de habilidades blandas necesarias para la práctica profesional (técnicas de litigación, estrategias de negociación y mediación, escritura legal, etcétera):

La expansión de la educación en habilidades profesionales ha preparado mejor a los estudiantes para representar a sus clientes, pero el currículo contiene una notable omisión: falla en enseñar a los estudiantes cómo ofrecer servicios en forma eficiente. En efecto, la mayoría de las escuelas de Derecho y la mayoría de los programas de clínica jurídica continúan enseñando un modelo de representación predominantemente basado en la figura del mandato, en el cual cada cliente recibe un trato altamente personalizado que demanda tiempo de dedicación exclusiva que resulta ser necesariamente caro (Perlman, 2019: 76). ${ }^{9}$

También es fundamental entender que las nuevas tecnologías de la información y las comunicaciones no sólo son herramientas útiles a la profesión tradicional de la abogacía, como alguno afirmó en su oportunidad (Volokh, 1996: 2.085), sino que es probable que ellas constituyan - en el mediano y largo plazo- la forma misma en que dicha profesión se ejercerá en el futuro (Susskind y Susskind, 2018: 125).

De momento, nada de esto parece estar del todo claro en la comunidad jurídica (académica y profesional). En tal sentido, incluso en Estados Unidos - como lo ha hecho notar con pesar Elizabeth Porter en la introducción de un extenso trabajo de investigación - se sigue enseñando el derecho y, en consecuencia, instruyendo a los futuros profesionales del área de la misma forma que se hacía hace más de cien años.

El derecho ha sido atrapado en una camisa de fuerza. La internet ha revolucionado los medios y las comunicaciones, reemplazando el texto con una vertiginosa variedad de gráficos multimedia e imágenes. Facebook alberga más de 150.000 millones de fotos. Las cortes gastan millones en juicios que involucran tecnología. Pero dichas innovaciones difícilmente han impactado en los escritos legales en blanco y negro. Los tratados legales continúan evocando a Blackstone y Kent; la mayoría de los libros de estudios de casos son facsímiles de Langdell; y las revistas legales recuerdan la Revista de Derecho de Harvard del año 1887. Ninguna de esas formas de transmisión del derecho ha hecho suyas - o siquiera notado-las normas modernas de saturación de imagen comunicacional. Litigantes, académicos y cortes han estado reutilizando los mismos moldes formalistas por más de un siglo. Esquemas que fue-

9. Eficiente, en el contexto en que Perlman lo emplea, no refiere simplemente a un criterio económico de rendimiento, sino a la posibilidad de utilizar recursos escasos para satisfacer demandas sociales urgentes: la provisión de servicios legales. Según Perlman, en Estados Unidos el costo excesivo de los servicios legales hace casi imposible a una parte significativa de la población acceder a la justicia, sobre todo en causas civiles. De ahí que la idea de ser eficiente en la realización de los servicios legales mediante la utilización de nuevas tecnologías no sólo redite desde el punto de vista económico, sino también satisfaga las exigencias mínimas éticas de un sistema legal: el acceso a la justicia (Perlman, 2019: 75). 
ron concebidos antes del uso extendido de la cámara, y ni qué decir del computador (Porter, 2014: 1687).

Si el diagnóstico hecho por algunos de estos teóricos resulta ser cierto, la forma tradicional de estudiar y ejercer la profesión de abogado quedará muy pronto obsoleta, pues las nuevas tecnologías, insistimos, no sólo son herramientas útiles para el ejercicio de las profesiones legales. Ahora bien, ¿qué tan consciente está el medio jurídico nacional de todo esto? Esta será la última cuestión que trataremos de dilucidar en este trabajo

\section{El estado de la cuestión en Chile: La aproximación didáctica con ocasión de las TIC y el trabajo dogmático-jurídico}

Antes hemos afirmado que existen, por lo menos, tres ámbitos en que las TIC e internet están transformando la profesión jurídica: la digitalización creciente de recursos y procedimientos jurídicos; la existencia de un mercado legal virtual y el uso generalizado de redes sociales como herramientas de la profesión legal, y el aumento progresivo de programas inteligentes en el ámbito del mercado legal. La cuestión es ver ahora si la academia nacional es sensible a estos cambios. Para resolver esta cuestión parece idóneo recurrir a la producción literaria de los últimos años. Obrando de este modo, podremos advertir qué tan interesados están los académicos nacionales en los cambios que están generando las TIC en el mundo del derecho y, de ser así, cuáles de estos cambios han recibido atención.

Nos parece que existen, a lo menos, tres formas en que las TIC pueden ser introducidas en el acervo cultural de los futuros abogados: en primer lugar, pueden ser $-\mathrm{y}$ de hecho lo son - utilizadas como recursos pedagógicos para la enseñanza y el aprendizaje del derecho (aulas virtuales, documentos compartidos, presentaciones electrónicas, clases vía streaming) y, eventualmente, esos recursos pueden servir como insumos para lo que más arriba hemos denominado un complemento al ejercicio profesional, cuestión que supondría una alfabetización digital efectiva. Desde una perspectiva dogmática, en segundo lugar, las tecnologías de la información y las comunicaciones pueden ser estudiadas como elementos que inciden en la transformación o adaptación de las formas jurídicas tradicionales (contratación, delitos y otros). En este caso podemos hablar de las TIC como un problema contemporáneo para las diferentes ramas del derecho que en el mundo angloparlante se vincula con la idea de law and technology. Por último, las TIC podrían ser analizadas como formas actuales - pero sobre todo futuras- de prestación de los servicios legales mismos. Aquí correspondería algún tipo de aproximación al conocimiento y diseño de aplicaciones, gestión y manejo de altos flujos de información, comoditización de servicios, etcétera. En este caso, se suele preferir la expresión legaltech. 


\section{La aproximación didáctica}

Las TIC, como decimos, pueden entrar en el campo formativo de los futuros profesionales del derecho a través de los recursos didácticos - en un sentido amplio-.$^{10}$ Sin embargo, es necesario en este punto hacer una distinción inicial. En efecto, es posible hablar de una suerte de "espacio tecnológico institucional», en el cual convergen los diversos programas de formación académica de una universidad a través de una plataforma común —usualmente denominada «aula virtual»—, que provee a sus usuarios recursos tecnológicos de carácter general (posibilidad de foros, archivos compartidos, edición multimedia y más). En algunas casas de estudio nacionales, dichos espacios tecnológicos llegan incluso al aula física en forma de conexión inalámbrica a la red, pizarras digitales, mesas interactivas, «clic keras» y otros. En el caso específico de las escuelas de Derecho chilenas, al formar parte de alguna universidad, es dable suponer que, de una forma más o menos intensa y más o menos provechosa, participan de ese espacio de formación virtual. Éste no es, con todo, el aspecto de las TIC que nos importa explorar en este trabajo.

Dejando de lado lo que aquí hemos denominado «espacio tecnológico institucional», cabría preguntarse si las TIC, por una parte, son en la actualidad recursos empleados de manera habitual en los programas de formación de los profesionales del derecho y, por otra, si sobre el particular se ha hecho algún tipo de investigación de campo que muestre la utilidad de tal estrategia. A esta cuestión la podríamos denominar el «factor de impacto de las TIC en la enseñanza y aprendizaje del derecho». Por último, también cabría la posibilidad de analizar el uso de las TIC en este nivel (didáctico) como herramientas que, diseñadas para el aprendizaje efectivo del derecho, podrían proyectar sus virtudes a la práctica profesional misma en tanto expresión de una alfabetización digital efectiva para la profesión. A este aspecto lo podríamos denominar «uso y desarrollo de nuevas tecnologías para la solución de problemas legales».

No es el propósito de este artículo hacer un análisis exhaustivo y concluyente del factor de impacto de las TIC en la enseñanza y aprendizaje del derecho y el uso y

10. Llevadas al ámbito de la enseñanza y aprendizaje, las TIC suelen denominarse TAC (tecnologías del aprendizaje y del conocimiento). Dentro de ellas es posible ubicar un conjunto de recursos materiales e informáticos, destacando por su importancia: las aulas virtuales (o espacios digitales de convergencia institucional); los programas multimediales de presentación de contenidos (Prezi, Emaze, Canva, Pixton); los programas de creación o edición de contenidos multimedia (Perfect Video, Youtube Editor, Screener); los algoritmos de trabajo colaborativo (Google Drive, Padlet); los programas de comunicación vía streaming (Zoom, Skype, Hangouts, ooVoo, CQPhone). Además de las TAC, el propósito con que se usan las nuevas tecnologías y las circunstancias singulares sus usuarios, han dado lugar a las llamadas TEP (tecnologías de empoderamiento y participación) y EPA (entornos personales de aprendizaje, abreviados también como PLE, por sus siglas en inglés). 
desarrollo de nuevas tecnologías para la solución de problemas legales. Sin embargo, sí podemos intentar - aunque sea a nivel de conjetura - ver si estos temas, por lo menos, están presentes en la discusión actual sobre la enseñanza y aprendizaje del derecho. En este sentido, una revista especializada de la Universidad de Chile nos puede ser de utilidad. Nos referimos a la Revista Pedagogía Universitaria y Didáctica del Derecho (RPUDD). ${ }^{11}$

Consultados todos los números disponibles en línea de la RPUDD, se puede advertir que sus colaboraciones, por lo menos hasta el primer semestre de 2020, se mantienen en un registro bastante conservador. Predominan, como es normal, los temas relativos a metodología, diseño curricular y didáctica. Hasta el 2016, entre sus numerosos e interesantes artículos, la cuestión de las TIC - salvo un par de colaboraciones referidas a recursos cinematográficos- está ausente de la discusión en los tres niveles que hemos propuesto de aproximación al tema (espacio tecnológico institucional, factor de impacto, y uso y desarrollo de TIC). Sin embargo, a partir del número 1 de 2017, se introduce en la revista una sección denominada «Innovación». Pensamos que dicha incorporación se debe a una necesidad que advierte el comité editorial de hacerse cargo de las nuevas prácticas académicas, dentro de las cuales habría que ubicar el uso de TIC. Ahora bien, resulta interesante que de todos los artículos que desde el 2017 han aparecido en dicha sección, sólo tres se hacen cargo del tema de las TIC en la enseñanza del derecho. También es interesante que de los únicos tres artículos - en todos los números de la revista - consagrados a la cuestión de las tecnologías, dos de ellos han sido escritos con ocasión de experiencias internacionales (Argentina y México). ${ }^{12}$ Con lo que, en resumen, la revista contiene un único artículo nacional sobre el tema que nos ocupa.

11. Revista Pedagogía Universitaria y Didáctica del Derecho, disponible en https://pedagogiaderecho. uchile.cl.

12. En el primero, Nidia Cicero expone con claridad y agudeza en su artículo «Innovar la enseñanza del derecho: ¿Sólo se trata de tecnologías de la información y comunicación?» tanto los riesgos que entraña el nuevo espacio tecnológico para la profesión legal, como los desafíos que las TAC suponen para los docentes y alumnos de Derecho. Su ensayo podríamos clasificarlo dentro de lo que hemos denominado «espacio tecnológico institucional e impacto de las TIC», referido en su caso al contexto de la Facultad de Derecho de la Universidad de Buenos Aires, Argentina (Cicero, 2018: 91-109). Cristina Cázares Sánchez, por su parte, en su trabajo titulado «Inclusión de las TIC y la enseñanza en la investigación jurídica en el posgrado en Derecho a nivel de especialidad de la UNAM», nos relata una experiencia concreta de trabajo colaborativo - sobre todo en la búsqueda coordinada de información pertinente para la realización de una investigación - en el ámbito del derecho empresarial. Dada la vinculación que en dicho texto se hace entre investigación colaborativa y derecho empresarial, es posible pensar que por lo menos aquellos estudiantes que participaron de dicho curso aprendieron a usar nuevas tecnologías en la generación de conocimiento y en la solución de problemas, cuestión que podría incluirse, aunque en un sentido más bien modesto, en lo que hemos denominado «uso y desarrollo de nuevas tecnologías para la solución de problemas legales» (Cázares Sánchez, 2017: 254-297). 
El artículo en cuestión explora las posibilidades que abren las TIC en la enseñanza del derecho para alumnos de los cursos introductorios. En esta dirección describe una experiencia particular - al parecer exitosa- de diseño y desarrollo de una actividad pedagógica en el contexto curso de introducción al derecho mediante la utilización de PLE (personal learning environment). Se trata, pues, de un trabajo que podríamos ubicar dentro de las cuestiones referidas al factor de impacto de las TIC en la enseñanza y aprendizaje del derecho. Tratándose, en todo caso, de una experiencia singular, no resulta fácil extraer muchas conclusiones sobre ella (Becerra Valdivia, 2017: 239-244).

En todo caso, pensamos que es sólo una cuestión de tiempo para que comiencen a aparecer en la Revista Pedagogía Universitaria y Didáctica del Derecho artículos que avancen en el estudio y comprensión de las TIC desde una perspectiva más amplia (uso y desarrollo de nuevas tecnologías para la solución de problemas legales).

Por último, nos parece adecuado destacar, a propósito de lo que hemos denominado «la aproximación pedagógica a las TIC», dos libros. El primero es Nuevos desafíos de la enseñanza del derecho en Chile (Scheechler, Aedo y Del Piano, 2013), obra colectiva que de alguna manera aborda la cuestión de las TIC en la enseñanza y aprendizaje del derecho, sobre todo en su capítulo dedicado a las plataformas virtuales para el aprendizaje colaborativo en el derecho internacional público. Aunque, al igual de lo que acontece con las publicaciones de la Revista Pedagogía Universitaria y Didáctica del Derecho, las referencias a las nuevas tecnologías están limitadas a las cuestiones sobre los espacios tecnológicos institucionales y los factores de impacto de las TIC en la enseñanza y aprendizaje del derecho. Por ello, aquí también hace falta algún tipo de reflexión teórica y práctica sobre el uso y eventual desarrollo de nuevas tecnologías como formas contemporáneas del ejercicio de la profesión legal. El otro libro es Nuevos paradigmas e innovaciones en la enseñanza del derecho (Becerra Valdivia, 2015). En esta obra encontramos una sola colaboración vinculada de manera directa a las TIC: «¿El buey o la carreta? Las TIC al servicio de la planificación de la enseñanza del derecho», de Christian Scheechler. Este trabajo, al igual que el ya comentado de Katherine Becerra, describe una experiencia particular en el uso de las TIC, pero desde la perspectiva de una planificación consciente de su uso y pertenencia con relación a los contenidos y objetivos de un curso de Derecho Penal. Como se aprecia, este artículo puede ser ubicado dentro de los temas referidos al factor de impacto de las TIC en la enseñanza y aprendizaje del derecho.

\section{La aproximación dogmática}

Respecto de la cuestión dogmática — es decir, el estudio del derecho frente a las TIC-, existe una literatura en aumento que, suponemos, se traduce en la incorporación de algún tipo de discusión en aula sobre el particular - a propósito del pro- 
ceso de formación de profesionales del derecho-- De hecho, el tema ha cobrado un grado de importancia tal en nuestro medio que, además de las revistas nacionales de derecho de carácter general, existen dos revistas especializadas: la Revista Chilena de Derecho y Tecnología y su predecesora Revista de Derecho Informático ${ }^{13}$-ambas dependientes de la Facultad de Derecho de la Universidad de Chile-, que reúnen artículos de primer nivel sobre los más variados ámbitos del derecho en que las TIC inciden o podrían incidir.

Ahora bien, cuando se analizan los índices de estas revistas - y de otras revistas nacionales de derecho de carácter general-, pronto se advierte un patrón bastante conservador en las líneas editoriales. En estas revistas, por lo menos hasta 2018, las cuestiones relativas a las nuevas tecnologías aparecen indisolublemente ligadas a los eventuales problemas jurídicos que ellas suscitan (law and technology); pero no existe por parte de los autores igual sensibilidad a las cuestiones relativas a la transformación de la práctica profesional a través de las nuevas tecnologías (legaltech), cuestión interesante si tenemos en cuenta la creciente importancia de este fenómeno. De hecho, no resulta muy difícil agrupar estos trabajos de derecho y tecnología en unos cuantos ejes temáticos bien definidos: protección de datos; derecho de autor y propiedad intelectual; nuevos problemas jurídicos a propósito de las TIC, y derecho penal y TIC.

A partir de 2019, sin embargo, aparecen en la Revista Chilena de Derecho y Tecnología dos interesantes estudios referidos a la tecnología legal y sus eventuales consecuencias para la profesión jurídica. Rodrigo Monteiro, en su artículo «Abogacía laboral 4.0: Inteligencia artificial y problemáticas profesionales en la abogacía brasileña», ofrece un excelente análisis de los problemas jurídicos y prácticos que supone el uso de inteligencia artificial para la solución de demandas jurídicas de alta sensibilidad. Aunque el artículo se concentra en la práctica empresarial de Hurst Capital Ltda. (Brasil), en particular en sus chatbot Valentina (materias laborales), Haroldo (cuestiones de consumo) y Leopoldo (el chatbot del contribuyente), el análisis es de más amplio calado. Como el autor nos indica, en la implementación de la inteligencia artificial a materias legales han de tenerse en consideración múltiples aspectos: legales, económicos y éticos. En estas tres dimensiones no se advierten suficientes garantías para los usuarios de estos servicios (chatbot), sino más bien algunos riesgos de que los usuarios vean disminuidos sus derechos de forma considerable (Monteiro Pessoa, 2019: 177-180).

El otro trabajo aparecido en la RCHDT en 2019, de autoría de Juan Luis Goldenberg, analiza el tema de herramientas de big data y fintech - específicamente el de asesoría financiera- para prevenir y aliviar el sobreendeudamiento del consumidor, a fin de hacer una propuesta actual tanto para el diseño normativo del mercado del

13. Descontinuada desde el año 2009. 
crédito como para ayudar a una planificación inteligente en los procedimientos de reestructuración de deuda. De acuerdo con Goldenberg, la posibilidad de usar la información disponible en el sistema, unido a estrategias financieras innovadoras derivadas del uso de tecnología, constituye una oportunidad de reestructuración y saneamiento del mercado del crédito.

La hipótesis con la que trabajamos es que estas herramientas tecnológicas, que disminuyen los costos de la formación y transmisión del consejo y de la advertencia al consumidor vulnerable, constituyen mecanismos idóneos para que las entidades crediticias puedan predecir con más precisión los riesgos de sobreendeudamiento, comunicarlos de manera adecuada al consumidor financiero e incluso ofrecer mecanismos de alivio una vez éste se encuentre en el área de la insolvencia y del sobreendeudamiento (Goldenberg, 2019: 4).

No cabe duda de que, dada la actual contingencia sanitaria mundial producida por el covid-19, ideas como las propuestas por Goldenberg podrían ser de invaluable utilidad tanto para el Gobierno como para las empresas financieras y los consumidores.

Estas investigaciones, inscritas en lo que más arriba — siguiendo a Susskind- hemos denominado «modelos paraprofesionales y de inteligencia incorporada en el sistema», constituyen la incipiente literatura jurídica sobre el particular que es posible hallar en las revistas nacionales especializadas de derecho. ${ }^{14}$ Probablemente esto se deba a que los académicos e investigadores que exponen los resultados de sus trabajos en tales revistas adoptan un enfoque bastante tradicional: las TIC, como cualquier otro elemento novedoso, presentan ciertos desafíos teóricos y prácticos — de interpretación y aplicación del derecho positivo- que se intentan superar mediante una adecuada comprensión de los textos normativos. De manera indirecta, pensamos, es probable que estos trabajos sean aprovechados por los alumnos de Derecho de pre y posgrado, en la medida en que son introducidos a modo ejemplar en el estudio y discusión de algunos temas particulares de dogmática jurídica. En este sentido, los futuros abogados -o profesionales del derecho, en el caso de los programas de posgrado- reciben alguna noticia de las TIC, aunque desde una perspectiva nominal y conservadora.

14. Este 2020 ha aparecido en el volumen 38 de la prestigiosa revista Computer Law \& Security Review otro artículo que avanza en la dirección de una reflexión en torno a las cuestiones de legaltech: hablamos de «Use of artificial intelligence by tax administrations: An analysis regarding taxpayers' rights in Latin American countries». Si bien se trata de una publicación extranjera, la autoría de este trabajo corresponde a Faúndez, Mellado y Aldunate (2020), investigadores del Núcleo de Derecho, Inteligencia Artificial y Tecnología (Derecho PUCV). En este trabajo se analiza la posible afectación de los derechos de los contribuyentes en virtud de la expansión del uso de la inteligencia artificial para la determinación de los tributos y la imposición de sanciones tributarias en diferentes países de la región. Una vez más, aquí estamos en presencia de un modelo mixto paraprofesional y de inteligencia incorporada en el sistema. 
Para encontrar en el medio nacional algún tipo de reflexión sobre las tecnologías de la información y las comunicaciones en un sentido más amplio —más allá de law and technology - , hay que acudir a otro tipo de publicaciones. Por una parte, es posible hallar algunos interesantes trabajos en la revista Cultura-Hombre-Sociedad (CUHSO) de la Universidad Católica de Temuco. Aunque, por desgracia, tampoco estas aproximaciones más generales a la cuestión se vinculan directamente con el derecho y la profesión jurídica, pues se limitan a un análisis de carácter más político institucional del impacto que tienen en la actualidad las TIC. ${ }^{15}$

Donde sí es posible encontrar, por lo menos de manera indirecta, un análisis sobre las implicancias de las nuevas tecnologías para el ejercicio de la profesión legal es en el trabajo de Velasco y Viollier, titulado Información financiera y discriminación laboral en Chile: Un caso de estudio sobre big data. En dicha obra se analiza, entre otras cosas, los aspectos dogmático-jurídicos y tecnológicos involucrados en los procesos de selección de personal a través de algoritmos y sus implicancias en materia laboral (principio de no discriminación). En tal sentido, la conclusión de estos autores en esta materia es categórica:

La adopción de herramientas de big data en los departamentos de recursos humanos de las empresas presenta un gran desafío regulatorio para nuestro país. El estudio del marco regulatorio nacional de protección de datos personales, las modificaciones que ha sufrido en los últimos quince años y los proyectos que buscan modernizarlo indican que nuestra legislación no está preparada para la adopción de herramientas de big data en los campos de selección de personas. La legislación laboral también parece insuficiente, ya que no otorga una protección adecuada ante la discriminación arbitraria a aquellas personas que están postulando a puestos de trabajo, pero que aún no han sido contratadas (Velasco y Viollier, 2016: 33).

Es el aspecto de la no discriminación en la selección de personal la vía por la que estos autores intuyen que la automatización informática de la selección de personal podría ser altamente complicada. Sobre todo, si se considera que la regulación laboral, que se ocupa de los problemas de discriminación, parece suponer una relación contractual en curso, aspecto no comprendido en los procesos de selección. Procesos, por cierto, que ya no requerirán necesariamente de profesionales competentes,

15. Entre los artículos de esta revista, cabe destacar: «Desafíos jurídico-políticos en el entorno digital» (Wong Ramírez, Rojas-Mora y Binimelis Espinoza, 2018: 8-11); «Voto electrónico en Chile: una evaluación desde una perspectiva técnica, jurídica y política» (Viollier y Riveros, 2018: 12-40); «Nelson Quichillao, Twitter y la lucha de clases» (Valdebenito y Knipp, 2018: 92-115); «Estrategias discursivas de las instituciones públicas en la web: Nuevas formas de comunicar las políticas públicas» (Martínez y Zukerfield, 2018: 41-66), y «La organización política ciudadana asistida por TIC, una aproximación sobre la influencia del "efecto red", la brecha digital y la brecha participativa en el contexto chileno" (Mellado, 2018: 67-91). 
sino de «paraprofesionales» operadores del software (acción de reemplazo laboral y no de complemento).

Como se aprecia, los autores nacionales han comenzado a hacerse cargo del fenómeno de las TIC de una manera cada vez más recurrente y persistente. Aunque, como lo hemos advertido, los análisis, de momento, se han concentrado en las cuestiones normativo-jurídicas (derecho y tecnología) y no en sus implicancias prácticas exorbitantes a la norma de derecho (tecnología legal). Prueba de ello es la literatura disponible, en uno y otro caso, en las revistas nacionales de derecho.

La aproximación teórico-práctica sobre diseño de aplicaciones, gestión y manejo de altos flujos de información, comoditización de servicios y más involucrados en las TIC

Como hemos tenido oportunidad de ver, por el momento, la academia se ha ocupado del tema de las tecnologías de la información y las comunicaciones desde una perspectiva pedagógica (las TIC como tecnologías del aprendizaje y el conocimiento) y dogmática (las TIC frente al derecho); pero no se ha hecho cargo de las implicancias laborales (complemento y sustitución funcional) que, por lo menos en teoría, formarán parte del futuro próximo de la profesión legal.

Por el contrario, los abogados que ejercen la profesión o que tienen un contacto mayor con el trabajo legal parecen estar cada vez más interesados en las implicancias laborales de las TIC para la profesión jurídica (cuestiones de legaltech). En este sentido, hemos encontrado cuatro interesantes columnas de opinión que tienen por objeto la cuestión de las nuevas tecnologías en el ámbito del ejercicio profesional. ${ }^{16}$ En ellas, con todo, no se advierte ningún futuro distópico para la profesión, sino más bien la necesidad de adaptar la enseñanza del derecho y el ejercicio profesional a los nuevos tiempos.

El factor que ha operado en la mayoría de estas columnas de opinión como elemento elicitante de la reflexión ha sido el informe McKinsey ${ }^{17}$ sobre el impacto de la automatización en el mercado laboral. A partir de este informe, algunos extraen en sus comunicaciones interesantes consecuencias, junto con compartir una visión no apocalíptica del futuro.

16. Enzo Canales, «Inteligencia artificial y el impacto en el ejercicio del derecho», $L W Y R, \mathbf{2 0 1 7}$, disponible en https://bit.ly/2Sml5Xc; Felipe Muñoz, «Inteligencia artifical y derecho: ¿El fin de los abogados?», El Mercurio, Legal, 31 de octubre de 2017, disponible en https://bit.ly/2ETMFrv; Carla Sánchez, «Inteligencia artificial, ¿un mundo sin abogados?», Capital, 20 de julio de 2017, disponible en https://bit. ly/2EWYqOo; Daniel Fajardo, «Webdox: El matrimonio entre derecho y tecnología», La Tercera, Pulso, 1 de diciembre de 2018, disponible en https://bit.ly/2GuAZMi.

17. «Un futuro que funciona: Automatización, empleo y productividad», McKinsey Global Institute, enero de 2017, disponible en https://mck.co/2GuVg4w. 
En cuanto a las cifras, y en lo que a la profesión legal toca, el referido informe resulta benigno con los abogados —en comparación con otras profesiones u ocupaciones-:

Respecto a la abogacía, los cambios no parecen ser tan dramáticos, ya que McKinsey estima que tan sólo el 23\% del tiempo de los abogados es automatizable, mientras que un estudio conjunto del Instituto Tecnológico de Massachusetts (MIT) y la Escuela de Leyes de la Universidad de Carolina del Norte concluye que tan sólo el 13\% de las labores de los abogados puede ser suplida por computadores. ${ }^{18}$

Sin embargo, eso no significa en modo alguno que las nuevas tecnologías, y en especial la inteligencia artificial, no vaya a colonizar en forma progresiva más espacios de trabajo legal. En tal dirección, ya hay en marcha proyectos que van más allá de la mera recopilación y gestión de grandes volúmenes de material legal:

Desde hace un tiempo IBM viene perfeccionando a Ross, un asistente legal basado en Watson, sistema informático de inteligencia artificial capaz de responder preguntas legales complejas realizadas de manera oral por un cliente. Con una capacidad gigantesca de procesar bases de datos - una versión de él puede hacerlo hasta con un millón de libros por segundo-, analiza un caso generando conclusiones que puedan ser usadas para elaborar respuestas concretas, citando las leyes aplicables. No es un simple procesador de información de alta velocidad, éste se autocorrige, detecta patrones, predice los resultados más probables en un escenario hipotético, procesa lenguaje jurídico, sortea argumentos lógicos y elabora contratos con las cláusulas más convenientes. Más recientemente encontramos también Jurimetria, un producto desarrollado sobre la plataforma Machine Learning de Google, que permite analizar la jurisprudencia de los tribunales y con ello predecir los resultados más probables que podrán seguir los casos que se presenten en un futuro. Con un sistema simple e intuitivo de indicadores gráficos, calcula, por ejemplo, la posible duración de un proceso o la probabilidad de que un recurso sea acogido, tiene también el potencial para comparar los criterios utilizados por cada tribunal y nos entrega el desempeño de estos, entre otros aspectos. ${ }^{19}$

En cualquier caso, ambos columnistas se muestran optimistas frente a las nuevas tecnologías, en tanto ven en ellas más bien elementos de complemento funcional que factores de reemplazo laboral. Sin embargo, sin perder el optimismo, otros advierten que su uso complementario supone una redefinición de las competencias de los futuros abogados. En tal sentido, el abogado sabedor de la ley, capaz de recitar de memoria extensos pasajes legales, parece tener sus días contados..$^{20}$ Mientras que las competencias de innovación, pensamiento no lineal e imaginación para adaptarse a las nuevas exigencias profesionales, parece, serán las habilidades y competencias

18. Canales, «Inteligencia...».

19. Muñoz, «Inteligencia...».

20. Sánchez, «Inteligencia...». 
deseadas en el nuevo entorno laboral. Un ejemplo de ello es el emprendimiento de Webdox. $^{21}$

Por lo anterior, aun cuando la academia todavía no se ha hecho cargo del fenómeno a nivel de reflexión científica, a nivel institucional las facultades y escuelas de Derecho han comenzado a mostrar un incipiente interés en los desarrollos tecnológicos que supongan diseño de aplicaciones, gestión y manejo de altos flujos de información y comoditización de servicios. De momento, en todo caso, se trata de unas cuantas iniciativas aisladas (no vinculadas a los programas de estudio de pre y posgrado), ${ }^{22}$ pero que es dable suponer en el mediano y largo plazo comenzarán a exhibir sus resultados en el medio nacional. Una vez que ello ocurra, creemos, será evidente la necesidad de articular estos centros de innovación tecnológica con los programas de estudio de derecho de pre y posgrado. ${ }^{23}$

De lo dicho hasta el momento hay, por lo menos, cuatro puntos que llaman la atención. En primer lugar, resulta sorprendente que las implicancias para la profesión legal de las nuevas tecnologías hayan sido desatendidas tanto por los investigadores interesados en los temas de docencia del derecho como por la doctrina, sobre todo si se tiene en cuenta la omnipresencia de las nuevas tecnologías en casi todas las áreas del quehacer nacional. En segundo lugar, esta falta de atención y reflexión sobre las TIC contrasta con lo que acontece en otras latitudes. En tercer lugar, es esta omisión por parte de los autores nacionales la que, en alguna medida, explica la nula presencia curricular de cursos obligatorios sobre estas materias en las escuelas de Derecho - tanto a nivel de pregrado como de posgrado- $y$, en aquellos casos en que existen cursos optativos o libres relacionados con las TIC, ellas reciben una consideración subordinada a los problemas de dogmática jurídica, o bien se presentan como herramientas útiles al trabajo colaborativo, pero nunca como potenciales insumos productivos del trabajo legal con aptitud de desplazamiento (reemplazo) laboral. Por último, resulta llamativo que los autores, por lo menos en esta materia, asuman un rol reactivo a los operadores jurídicos que, de momento, parecen más sensibles a las oportunidades y amenazas de las TIC.

21. Fajardo, «Webdox». El sitio web del software es http://www.webdox.cl.

22. En nuestro medio nacional existen hoy tres centros de innovación e investigación tecnológica asociadas al derecho. Estos son el Instituto Chileno de Derecho y Tecnologías (http://www.icdt.cl); el Centro de Estudios en Derecho Informático (http://www.derecho.uchile.cl/cedi); y el recientemente creado Núcleo de Derecho, Inteligencia Artificial y Tecnología (Derecho PUCV), que por el momento no cuenta con sitio web ni información básica.

23. Fenómeno que ya se advierte en otros países. Sólo a modo ilustrativo, en el sitio web de Lawstudies, disponible en https://www.lawstudies.com/LLM/Technology-Law/, se pueden encontrar quince de los más prestigiosos programas académicos asociados a centros de innovación tecnológica de algunas de las universidades líderes en esta materia. 


\section{Conclusiones}

Aunque a lo largo de este trabajo hemos adelantado ciertas ideas generales, tal vez convenga en este momento extraer algunas conclusiones sobre el impacto que las TIC, eventualmente, tendrán para la profesión legal en el mediano y largo plazo.

En primer lugar, parece bastante evidente que hoy, a diferencia de lo que ocurría sólo hace algunos años, cierta alfabetización digital es necesaria. Sin embargo, por lo menos en Chile, no está claro en qué parte del periodo formativo de los futuros profesionales del derecho dicho conocimiento se adquiere. Las escuelas de Derecho, que continúan fieles a sus programas tradicionales y las oficinas de abogados, en su inmensa mayoría tampoco ofrecen un espacio idóneo para aprender sobre tecnología legal. En la práctica, esto se traduce en que el aprendizaje, uso e innovación de las TIC queda entregado por completo al denominado aprendizaje informal motivado por las inquietudes particulares de cada cual.

En segundo lugar, nos parece claro - por todo lo que hemos dicho-, que las tecnologías de la información y comunicaciones no deben seguir siendo consideradas tan sólo herramientas para el ejercicio profesional, sino que están transformando la profesión jurídica misma, sin que los académicos nacionales parezcan advertir los signos visibles de este fenómeno. En esta dirección, ellas ofrecen a los profesionales del derecho oportunidades de innovación, pero también representan amenazas de desplazamiento del trabajo rutinario jurídico del abogado mediante la automatización de procesos.

En tercer lugar, hay que entender que las tecnologías actuales son una nueva forma de llevar a cabo el trabajo legal y en tal sentido, por una parte, no se puede independizar la formación dogmática-jurídica de la competencial tecnológica. Por otra parte, tampoco las escuelas de Derecho pueden seguir pretendiendo que la alfabetización digital para la profesión legal será adquirida en «el espacio tecnológico institucional».

Por desgracia, en último lugar, pese a todo lo que hemos dicho y a lo evidente que pueden resultar algunas de nuestras afirmaciones, no resulta claro que las TIC vayan a recibir en el corto plazo mayor atención por parte de la academia. Cuando hablamos aquí de una «mayor atención» a las TIC, no nos referimos sólo a un conocimiento particular sobre éstas y sus usos, sino a la nueva forma de pensar asociada a ellas; forma de pensar que, por cierto, obligaría a revisar tanto los actuales programas de estudio de las escuelas de Derecho como la forma en que se enseñan y la manera en que los servicios legales se prestan.

Ahora bien, las razones sustantivas que explican esta reluctancia de la academia a las nuevas tecnologías, así como las posibles reformas curriculares y metodológicas que ellas demandan, son cuestiones en las cuales habría que detenerse con detalle. Pero dicha empresa, por cierto, escapa a esta investigación que simplemente ha querido poner en discusión, de la forma más ordenada posible, el estado actual de la cuestión en Chile. 


\section{Referencias}

Acosta, Ramón, Elizabeth Miquilena y Víctor Riveros (2014). «La infraestructura de la información y comunicación como mediadoras y el aprendizaje de la biología». Telos, 16 (1): 11-30. Disponible en https://bit.ly/34yRSoX.

Bauman, Zygmunt (2006). Liquid modernity. Padstow: Polity Press.

-. (2011). Daños colaterales. Buenos Aires: Fondo de Cultura Económica.

BeCerra Valdivia, Katherine (coordinadora) (2015). Nuevos paradigmas e innovaciones en la enseñanza del derecho. Santiago: Jurídicas de Santiago.

-. (2017). «El uso de los entornos personales de aprendizaje (PLE) en los cursos de introducción al derecho». Revista Pedagogía Universitaria y Didáctica del Derecho, 4 (1): 225-253. DOI: $10.5354 / 0719-5885.2017 .46255$.

Castells, Manuel (2005). La era de la información: Economía, sociedad y cultura. Volumen 1. Madrid: Alianza.

Cázares SÁnChez, Cristina (2017). «Inclusión de las TIC y la enseñanza en la investigación jurídica en el posgrado en derecho a nivel de especialidad de la UNAM». Revista Pedagogía Universitaria y Didáctica del Derecho, 4 (1): 254-297. DOI: 10.5354/0719-5885.2017.46256.

Chen, Min, Shiwen Mao, Yin Zhang y Víctor Leung (2014). Big data: Related technologies, challenges and future prospects. Nueva York: Springer.

Cicero, Nidia (2018). «Innovar la enseñanza del derecho: ¿Sólo se trata de tecnologías de la información y comunicación?». Revista Pedagogía Universitaria y Didáctica del Derecho, 5 (2): 91-109. DOI: 10.5354/0719-5885.2018.51976.

Delgado, Ana e Ignasi Beltrán (coordinadores) (2018). Derecho y TIC: Últimas innovaciones docentes. Barcelona: Huygens.

ECheVerri, Daniel (2016). Deep Web: TOR, FreeNet \& I2P. Privacidad y anonimato. Madrid: Zeroxword Computing.

EDWARDs, Lilian y Charlotte Waelde (editores) (2000). Law \& the internet. Londres: Hart.

FAúndez, Antonio, Rafael Mellado y Eduardo Aldunate (2020). «Use of artificial intelligence by tax administrations: An analysis regarding taxpayers' rights in Latin American countries». Computer Law \& Security Review, 38: 105441. DOI: 10.1016/j.clsr.2020.105441.

GoldenberG, Juan Luis (2019), «Herramientas del big data y fintech para prevenir y aliviar el sobreendeudamiento del consumidor: Una propuesta». Revista Chilena de Derecho y Tecnología, 8 (2): 5-32. DOI: 10.5354/0719-2584.2019.54051.

GutwiRTH, Serge, Ronald Leenes y Paul de Hert (editores) (2016). Data protection on move. Dordrecht: Springer.

HAN, Byung-Chul (2014). En el enjambre. Barcelona: Herder.

-. (2015a). La sociedad del cansancio. Barcelona: Herder. 
-. (2015b). Psicopolítica. Barcelona: Herder.

Harari, Yuval Noah (2016). Homo deus: Breve historia del mañana. Santiago: Debate. Horne, Dillon (2014). "Cloud computing, virtual law firms, and the legal profession». Cornell Law School Graduate Student Papers, 29. Disponible en https://bit. ly/3onUJsb.

LAmbert, Paul (2015). Gringras: The laws of the internet. Londres: Bloomsbury Professionals.

Larrosa, Juan Manuel (2000). «Enmiendas a la ley de Metcalfe». Disponible en https://bit.ly/2HOo8SN.

LARROUCAU, Jorge (2017). "Adiós a las fojas: Reglas procesales, autos acordados y tramitación electrónica en Chile». Revista de Derecho Privado, 33: 195-234. DOI: 10.18601/01234366.n33.08.

Lim, Yee Fen (2002). Cyberspace law. Melbourne: Oxford University Press.

Martínez, Maite y Gilda Zukerfield (2018). «Estrategias discursivas de las instituciones públicas en la web: Nuevas formas de comunicar las políticas públicas». Cultura-Hombre-Sociedad, 28 (2): 41-66. DOI: 10.7770/o719-2789.2018.cuhso.04.ao1.

Mellado, Alejandro (2018). «La organización política ciudadana asistida por TIC, una aproximación sobre la influencia del "efecto red", la brecha digital y la brecha participativa en el contexto chileno». Cultura-Hombre-Sociedad, 28 (2): 67-91. DOI: 10.7770/0719-2789.2018.cuhso.06.a04.

Metzner-SzIgeth, Andreas (2006). «El movimiento y la matriz-Internet y la transformación socio-cultural». Revista Iberoamericana de Ciencia, Tecnología, Sociedad e Innovación, 7. Disponible en https://bit.ly/3cRcHs1.

Monteiro Pessoa, Rodrigo (2019), «Abogacía laboral 4.o: Inteligencia artificial y problemáticas profesionales en la abogacía brasileña». Revista Chilena de Derecho y Tecnología, 8 (1): 167-183. DOI: 10.5354/0719-2584.2019.51276.

Moore, Gordon E. (1965). «Cramming more components onto integrated circuits». Electronics, 38 (8): 114-117.

-. (2006). «Moore’s law at 40». En David C. Brock (editor), Understanding Moore’s law. Pennsylvania: Heritage Press.

Patiño Builes, Albeiro (2014). «De la paradoja de la productividad y la ley de Moore al papel de las TIC en el aumento de la productividad de las empresas y de las naciones». Inge $\mathrm{CuC}$, 10 (2): 51-59. Disponible en https://bit.ly/30qLlE2.

Perlman, Andrew (2019). "The public's unmet need for legal services \& what law schools can do about it». Daedalus: The Journal of the American Academy of Arts \& Sciencies, 148 (1): 75-81. DOI: 10.1162/daed_a_00537.

Perlman, Andrew y Gabriel Tanenbaum (2016). «Thinking like a lawyer in the 21st century». Practice Innovations, 17 (1): 1-4. Disponible en https://tmsnrt.rs/3neKdof.

Phillips, Jeremy (editor) (2013). E-commerce and information technology law: Handbook. Londres: Butterworths. 
Porter, Elizabeth (2014). «Taking images seriously». Columbia Law Review, 114 (7): 1.687-1.782. Disponible en https://bit.ly/3lghkzz.

ReED, Chris y John Angel (editores) (2003). Computer law. Oxford: Oxford University Press.

Rincón, Fernado (2018). Derecho en la era de las TIC. Bogotá: Ecoe.

RostaIn, Tanina, Roger Skalbeck y Kevin G. Mulcahy (2013). «Thinking like a lawyer, designing like and architect: Preparing students for the 21st Century practice». Chicago-Kent Law Review, 88 (3): 743-755. Disponible en https://bit.ly/34n3SlZ.

Rowland, Diane, Uta Kohl y Andrew Charlesworth (2016). Information technology law. Abingdon: Routledge.

SCHALLER, Robert R. (1997). «Moore's law: Past, present, and future». IEEE Spectrum, 34 (6): 52-59. DOI: 10.1109/6.591665.

Scheechler, Christian, Cristian Aedo y Cristian del Piano (2013). Nuevos desafíos de la enseñanza del derecho en Chile. Santiago: Libromar.

Susskind, Richard (1996). The future of law. Oxford: Oxford University Press.

-. (2008). The end of Lawyers? Oxford: Oxford University Press.

-. (2013). Tomorrow's lawyers. Oxford: Oxford University Press.

Susskind, Richard y Daniel Susskind (2015). The future of the professions. Oxford: Oxford University Press.

-. (2018). «The future of the professions». Proceedings of the American Philosophical Society, 162 (2): 125-138. Disponible en https://bit.ly/3otqy2E.

VAldebenito, Jorge y Rocío Knipp (2018). «Nelson Quichillao, Twitter y la lucha de clases». Cultura-Hombre-Sociedad, 28 (2): 92-115. DOI: 10.7770/0719-2789.2018. cuhso.06.a05.

Velasco, Patricio y Pablo Viollier (2016). Información financiera y discriminación laboral en Chile: Un caso de estudio sobre big data. Santiago: Derechos Digitales. Disponible en https://bit.ly/3nf32QX.

Viollier, Pablo y Bastián Riveros (2018). «Voto electrónico en Chile: Una evaluación desde una perspectiva técnica, jurídica y política». Cultura-Hombre-Sociedad, 28 (2): 12-40. DOI: 10.7770/0719-2789.2018.cuhso.06.ao2.

Volokh, Eugen (1996). «Computer media for the legal profession». Michigan Law Review, 94 (6): 2.058-2.087. Disponible en https://bit.ly/3lb3NZw.

Wong Ramírez, Sulan, Julio Rojas-Mora y Helder Binimelis Espinoza (2018). «Desafíos jurídico-políticos en el entorno digital». Cultura-Hombre-Sociedad, 28 (2): 8-11. DOI: 10.7770/cuhso-V28N2-art1854.

Yoo, Christopher S. (2015). «Moore's law, Metcalfe's law, and the theory of optimal interoperability». Faculty Scholarship, 1.651. Disponible en https://bit.ly/3lgiZGl.

ZuKerfeld, Mariano (2015). «La tecnología en general, las digitales en particular: Vida, milagros y familia de la "ley de Moore"». Hipertextos, 4 (2): 87-115. Disponible en https://bit.ly/2HVoMhD. 


\section{Sobre el autor}

Johann S. Benfeld E. es doctor en Derecho por la Universidad de Salamanca, España. Profesor de Teoría y Filosofía del Derecho en la Escuela de Derecho de la Pontificia Universidad Católica de Valparaíso, Chile. Investigador del Núcleo de Derecho, Inteligencia Artificial y Tecnología (Derecho PUCV). Su correo electrónico es johann.benfeld@pucv.cl. (D) https://orcid.org/oooo-0oo1-6109-3878. 


\title{
REVISTA CHILENA DE DERECHO Y TECNOLOGÍA
}

La Revista de Chilena de Derecho y Tecnología es una publicación académica semestral del Centro de Estudios en Derecho Informático de la Facultad de Derecho de la Universidad de Chile, que tiene por objeto difundir en la comunidad jurídica los elementos necesarios para analizar y comprender los alcances y efectos que el desarrollo tecnológico y cultural han producido en la sociedad, especialmente su impacto en la ciencia jurídica.

\author{
EDITOR GENERAL \\ Daniel Álvarez Valenzuela \\ (dalvarez@derecho.uchile.cl) \\ SITIO WEB \\ rchdt.uchile.cl \\ CORREO ELECTRÓNICO \\ rchdt@derecho.uchile.cl \\ LICENCIA DE ESTE ARTÍCULO \\ Creative Commons Atribución Compartir Igual 4.o Internacional
}

\begin{abstract}
La edición de textos, el diseño editorial
y la conversión a formatos electrónicos de este artículo

estuvieron a cargo de Tipográfica

(www.tipografica.io).
\end{abstract}

